\title{
Geometry of Valley Growth
}

\author{
By A.P. PETROFF ${ }^{1}$, O. DEVAUCHELLE ${ }^{1}$, D. M. A B R A M S $\mathbf{S}^{1,2}$, \\ A. E. L O B K OVSKY ${ }^{1}, A$. KUDROLLI I D . H ROTHMA N ${ }^{1}$ \\ ${ }^{1}$ Department of Earth, Atmospheric and Planetary Sciences, Massachusetts Institute of \\ Technology, Cambridge, MA 02139 USA \\ ${ }^{2}$ Present address: Department of Engineering Sciences and Applied Mathematics, \\ Northwestern University, Evanston, IL 60208 \\ ${ }^{3}$ Department of Physics, Clark University, Worcester, MA 01610
}

(Received 31 October 2018)

\begin{abstract}
Although amphitheater-shaped valley heads can be cut by groundwater flows emerging from springs, recent geological evidence suggests that other processes may also produce similar features, thus confounding the interpretations of such valley heads on Earth and Mars. To better understand the origin of this topographic form we combine field observations, laboratory experiments, analysis of a high-resolution topographic map, and mathematical theory to quantitatively characterize a class of physical phenomena that produce amphitheater-shaped heads. The resulting geometric growth equation accurately predicts the shape of decimeter-wide channels in laboratory experiments, 100-meter wide valleys in Florida and Idaho, and kilometer wide valleys on Mars. We find that whenever the processes shaping a landscape favor the growth of sharply protruding features, channels develop amphitheater-shaped heads with an aspect ratio of $\pi$.
\end{abstract}

\section{Introduction}

When groundwater emerges from a spring with sufficient intensity to remove sediment, it carves a valley into the landscape (Dunne 1980). Over time, this "seepage erosion" causes the spring to migrate, resulting in an advancing valley head with a characteristic rounded form (Lamb et al. 2006). Proposed examples of such "seepage channels" include centimeter-scale rills on beaches and levees (Higgins 1982; Schorghofer et al. 2004), hundred-meter-scale valleys on Earth (Schumm et al. 1995; Abrams et al. 2009; Russell 1902; Orange et al. 1994; Wentworth 1928; Laity \& Malin 1985), and kilometer-scale valleys on Mars (Higgins 1982; Malin \& Carr 1999; Sharp \& Malin 1975). Although it has long been thought that the presence of an amphitheater-shaped head is diagnostic of seepage erosion (Higgins 1982; Russell 1902; Laity \& Malin 1985), recent work suggests that overland flow can produce similar features (Lamb et al. 2006, 2008). To address this ambiguity, we seek a general characterization of processes that produce channels indistinguishable in shape from seepage channels.

We first identify the the interface dynamics (Brower et al. 1983; Ben-Jacob et al. 1983; Kessler et al. 1985; Shraiman \& Bensimon 1984; Marsili et al. 1996; Pelcé 1988, 2004) appropriate for amphitheater-shaped valley heads formed by seepage erosion. We then show that the same dynamics apply in a more general setting. We find that whenever the processes shaping a landscape cause valleys to grow at a rate proportional to their curvature, they develop amphitheater-shaped heads with a precise shape. This result clarifies the controversy surrounding terrestrial and Martian valleys by showing that 

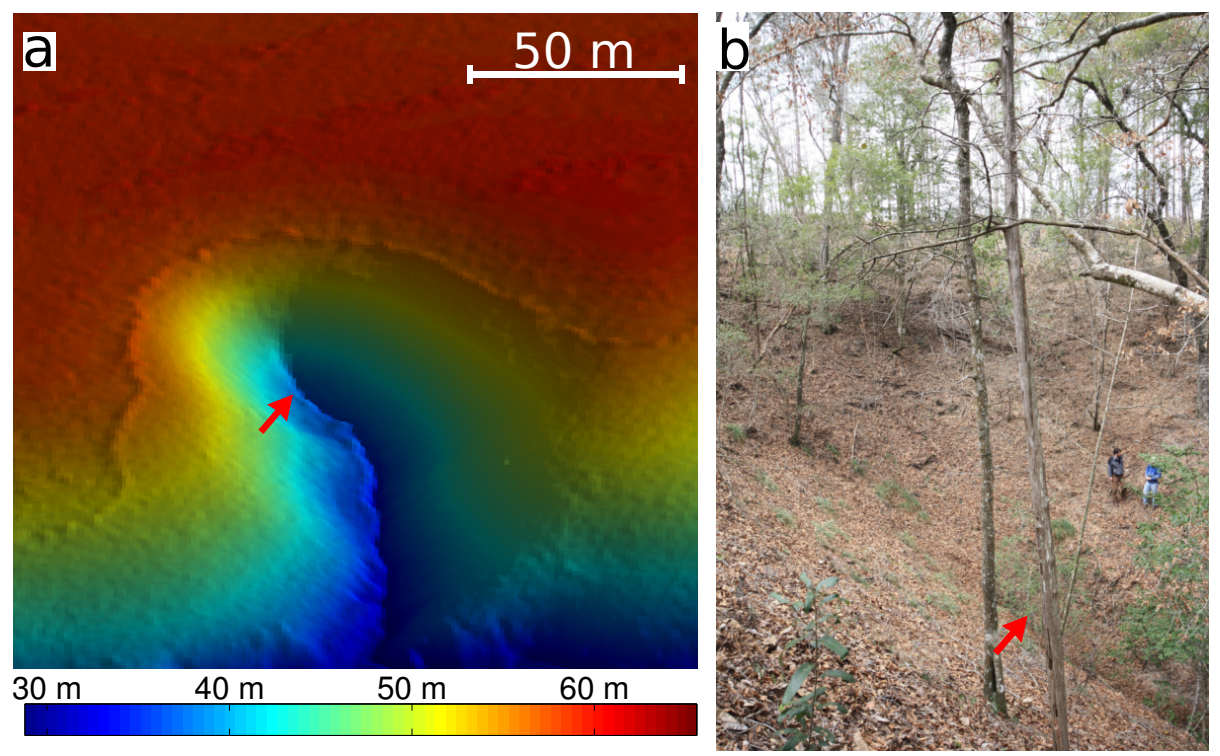

FiguRE 1. Examples of seepage valleys from the Florida network. (a) Topography obtained from a high-resolution map (Abrams et al. 2009) showing the rounded "amphitheater-shaped" valley head surrounding a spring (red arrow). Colors represent elevation above sea level. (b) A seepage valley head as viewed from the side wall. The red arrow shows the approximate position of the spring. Note people for scale.

many of these features are quantitatively consistent with a class of dynamics which includes, but is not limited to, seepage erosion.

\section{The Florida network}

To provide a precise context for our analysis, we first focus on a well-characterized kilometer-scale network of seepage valleys on the Florida panhandle (Schumm et al. 1995; Abrams et al. 2009) (figure 1). This network is cut approximately $30 \mathrm{~m}$ into homogeneous, unconsolidated sand (Schumm et al. 1995; Abrams et al. 2009). Because the mean rainfall rate $P$ is small compared to the hydraulic conductivity of the sand, nearly all water enters the channel through the subsurface (Schumm et al. 1995; Abrams et al. 2009). Furthermore, sand grains can be seen moving in streams near the heads, implying that the water drained by a spring is sufficient to remove sediment from the head. Finally, a myriad of amphitheater-shaped valley heads $(n>100)$ allows for predictions to be tested in many different conditions.

We begin by finding the equilibrium shape of the water table in the Florida valley network. This shape describes how water is distributed between different heads. When the groundwater flux has a small vertical component (relative to the horizontal components), the Dupuit approximation (Bear 1979) of hydrology relates the variations in the height $h$ of the water table above an impermeable layer (Schumm et al. 1995; Abrams et al. 2009 ) to the mean rainfall rate $P$ and the hydraulic conductivity $K$ through the Poisson equation

$$
\frac{K}{2} \nabla^{2} h^{2}+P=0
$$

To simplify our analysis, we define two rescaled quantities: the Poisson elevation $\phi=$ $(K / 2 P)^{1 / 2} h$ and the Poisson flux $q_{p}=\left\|\nabla \phi^{2}\right\|$. The Poisson elevation is determined 
entirely from the shape of the network and, consequently, can be measured from a map without knowledge of $P$ or $K$. Physically, $q_{p}$ is the area that is drained by a small piece of the network per unit arc length. It is therefore a local version of the inverse drainage density (i.e., the basin area divided by total channel length). By construction, the groundwater flux $q=P q_{p}$. This measure of the flux differs from the geometric drainage area (Abrams et al. 2009) in that it is found from a solution of the Poisson equation, rather than approximated as the nearest contributing area.

Fig. 2a shows the solution of equation (2.1) around the valley network (supplementary material). Because the variations in the elevation at which the water table emerges are small $(\sim 10 \mathrm{~m})$ relative to the scale of the network $(\sim 1000 \mathrm{~m})$, we approximate the network boundary with an elevation contour extracted from a high resolution topographic map (Abrams et al. 2009) on which $\phi$ is constant (see supplementary material). For a specified precipitation rate, this result predicts the flux $q$ of water into each piece of the network.

To test this model of water flow, we compared the solution of equation (2.1) to measurements at 82 points in the network. Given a reported mean rainfall rate of $P=5 \times 10^{-8} \mathrm{~m} \mathrm{sec}^{-1}$ (Abrams et al. 2009), we find good agreement between observation and theory (Fig. 2b), indicating that equation (2.1) accurately describes the competition for groundwater. Additionally, we find that the water table elevation $h$ is consistent with a ground penetrating radar survey (Abrams et al. 2009) of the area (see supplementary material). To understand how the distribution of groundwater through the network produces channels with amphitheater-shaped heads, we proceed to relate the flux of water into a valley head to the geometry of the head.

\section{Relation of flux and geometry}

For an arbitrary network, there is no simple relationship between the flux of water into part of the network and its local shape. As each tip competes with every other part of the network, one can only find the local flux by solving equation (2.1). However, as first identified by Dunne (Dunne 1980), valleys cut by seepage grow when sections of the valley which protrude outwards (high positive curvature) draw large fluxes while indented sections (negative curvature) of the network are shielded by the network. Motivated by this insight, we seek the relationship between the flux into a piece of a valley network and its planform curvature. Fig. 2c shows that this relationship is consistent with a hyperbolic dependence of the Poisson flux (and hence the water flux) on the curvature. Consequently, at tips, where the curvature is high, this relationship can be approximated by the asymptote. Thus,

$$
q_{p} \simeq \Omega \kappa,
$$

where $\Omega$ is a proportionality constant related to the area drained by a single head. Thus we find a local relationship between the processes shaping a seepage valley, represented by the flux $q_{p}$, and the local geometry, represented by the curvature $\kappa$. We note that this relation may be further justified by a scaling argument (supplementary material), but here we merely employ it as an empirical observation.

\section{Geometric growth law}

In what follows we first ask how the flux-curvature relation (3.1) is manifested in the shape of a single valley head. To do so we first find the shape of a valley head that is consistent with the observed proportionality between groundwater flux and curvature. 

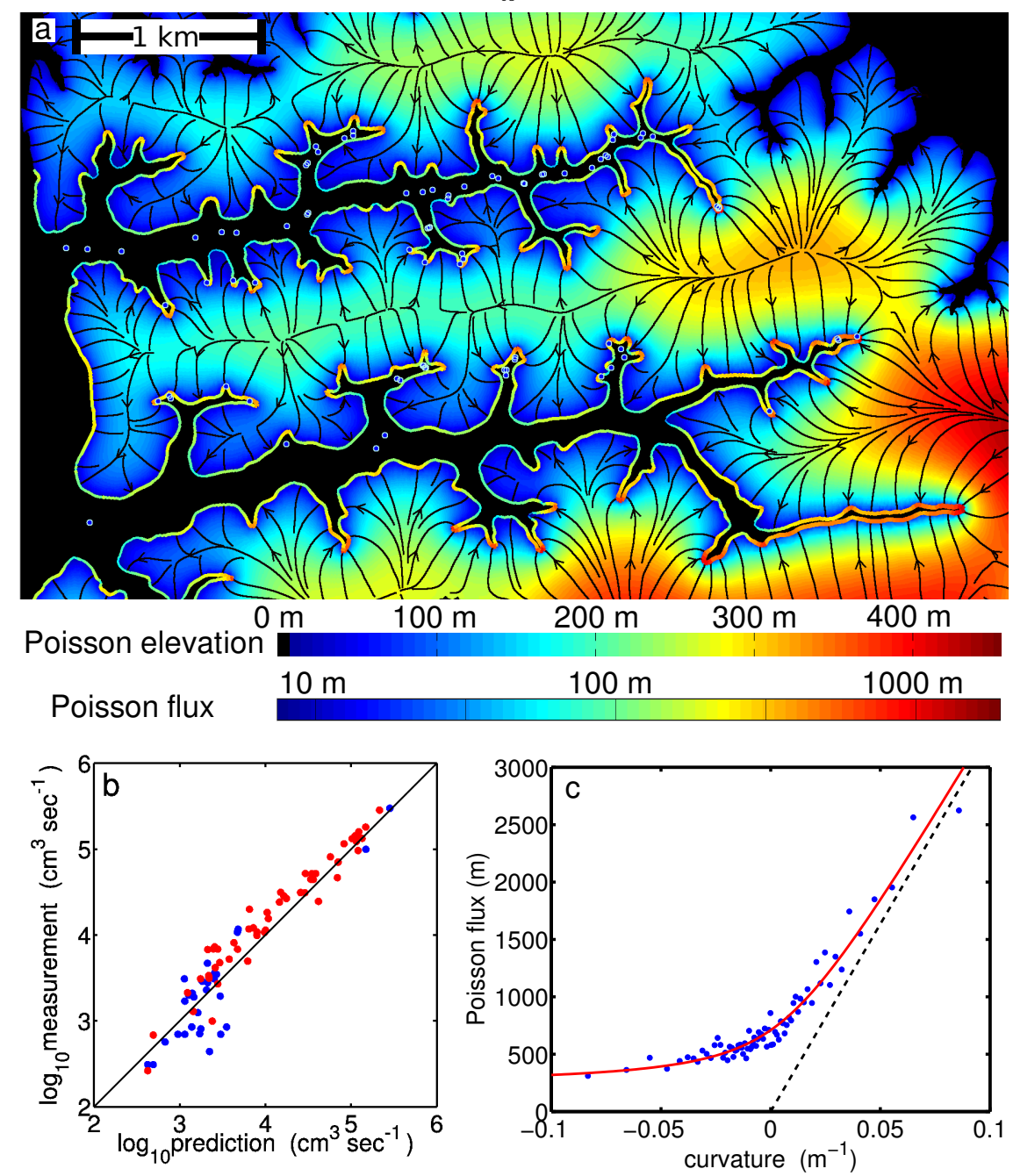

FiguRE 2. The water table and associated groundwater flux in the Florida network. (a) The magnitude of the Poisson flux (color intensity on boundary) is the size of the area draining into a section of the network per unit length. It is found by solving equation (2.1) around the channels as approximated with an elevation contour. Flow lines are in black. The water discharge was measured at blue circles. The Poisson elevation and Poisson flux are proportional to the water table height and groundwater flux, respectively. (b) Comparison of the predicted discharge to measurements at 30 points in network taken in January of 2009 (blue points) and 52 points in April of 2009 (red points). The black line indicates equality. This comparison is direct and requires no adjustable parameters. (c) We observe a hyperbolic relationship between the curvature of the valley walls and the predicted flux (red curve). In regions of high curvature (i.e. valley heads) the flux is proportional to curvature (dashed line).

This derivation relies on three steps. First, equation (3.1) is converted, with an additional assumption, into a relationship between the rate at which a valley grows outward and its planform curvature. Next, we restrict our attention to valley heads that grow forward without changing shape. This condition imposes a geometric relationship between growth and orientation. Combining these, we find a relationship between curvature and orientation that uniquely specifies the shape of a valley growing forward due to groundwater flow. Finally, we find that our theoretical prediction is consistent both with valleys cut by 
seepage and systems in which seepage is doubtful. This result leads us to conclude that seepage valleys belong to a class of systems defined by a specific relationship between growth and curvature which includes seepage erosion as a particular case.

Following past work (Howard 1988; Abrams et al. 2009), we assume that the amount of sediment removed from the head is proportional to the flux of groundwater; and thus, by equation 3.1, $\Omega \kappa$. From equation (3.1), the speed $c$ at which a valley grows outward is therefore proportional to the planform valley curvature $\kappa$. Setting $H$ equal to the difference in elevation between the spring and the topography it is incising, the sediment flux is

$$
H c=\alpha \Omega \kappa,
$$

where $\alpha$ is a proportionality constant with units of velocity. Equation (4.1) states that the more sharply a valley wall is curved into the drainage basin, the faster it will grow. The growth of the channel head is therefore "curvature-driven" (Brower et al. 1983).

This derivation of equation 4.1 marks a shift of focus from the mechanics that shape a seepage valley to the dynamics by which it evolves. Although the specific processes of groundwater flow and sediment transport have not been addressed explicitly, this generalization has two advantages. First, equation 4.1 is purely geometric and can be solved to provide a quantitative prediction for the shape of a valley head. Equally importantly, the generality of these dynamics suggests that the class of processes they describe may extend beyond seepage valleys and thus provide a quantitative prediction for the evolution of a wider class of channelization phenomena.

\section{Shape of a valley head}

We restrict our attention to steady-state valley growth. When the channel grows forward at a speed $c_{0}$ without changing shape, the outward growth balances the growth forward. If $\theta$ is the angle between the normal vector and the direction the channel is growing (Fig. 3), then $c=c_{0} \cos \theta$. Substituting this condition for translational growth into equation 4.1 relates the orientation of a point on the channel to the curvature at that point:

$$
\cos \theta=\frac{\alpha \Omega}{c_{0} H} \kappa(\theta),
$$

where $\kappa(\theta)$ denotes the dependence of curvature on orientation. Solving this equation (see appendix) for the shape of the curve with this property gives (Brower et al. 1983)

$$
y(x)=\frac{w}{\pi} \log \cos \left(\pi \frac{x}{w}\right),
$$

where $w=\pi \alpha \Omega /\left(c_{0} H\right)$ is the valley width and $\theta=\pi x / w$. The planform shape $y(x)$ is shown in Fig. 3. A notable feature of this solution is that all geometric aspects of the channel head are set by the absolute scale of the valley (i.e. the valley width). In particular, it follows from equations (5.1) and (5.2) that all seepage channels have a characteristic aspect ratio

$$
\frac{w}{r}=\pi,
$$

where $r$ is the radius of curvature of the tip (Fig. 3). By contrast, a semi-circular valley head, in which $w=2 r$, has an aspect ratio of 2 . 


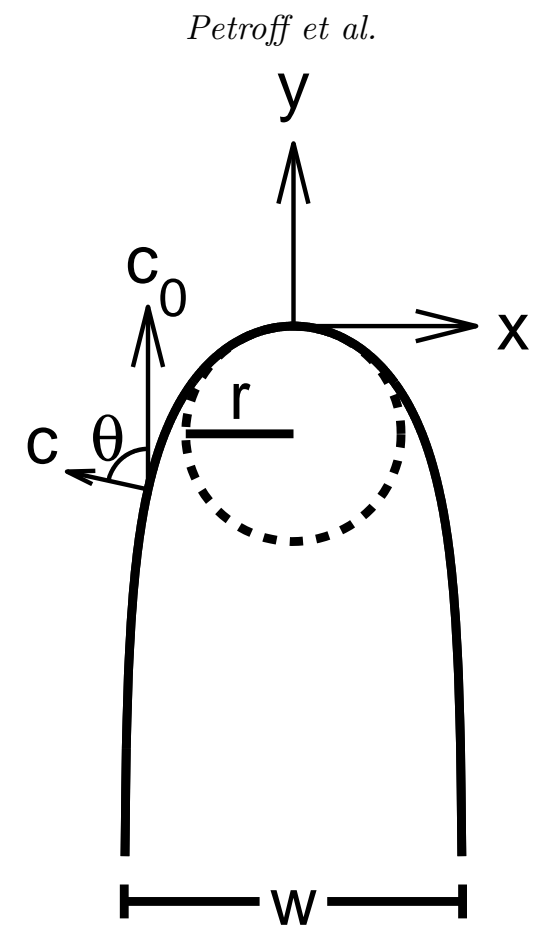

FiguRE 3. A balance between curvature-driven growth and translational growth sets the shape (eq. 5.2) of an amphitheater-shaped valley head (solid black curve). When a curve evolves due to curvature-driven growth, the normal velocity $c$ is inversely proportional to the radius of the best fitting circle at that point. When a curve translates forward, there is a geometric relationship between the speed at which a point translates $c_{0}$ and the speed at which it grows in the normal direction $c$. This balance produces channels with a well defined width $w$ and an aspect ratio of $w / r=\pi$.

\section{Comparison to observation}

To test these predictions, we first compare the shape of elevation contours extracted from 17 isolated, growing tips in the Florida network to equations (5.2) and (5.3). As these valley heads vary in size, a sensible comparison of their shapes requires rescaling each channel to the same size; we therefore non-dimensionalize each curve by its typical radius $w / 2$. To remove any ambiguity in the position where the width is measured, $w$ is treated as a parameter and is fit from the shape of each valley head. Fig. 4a compares all 17 rescaled channels heads to equation (5.2). Although each individual valley head may deviate from the idealization, the average shape of all valley heads fits the model well.

This correspondence between theory and observation is further demonstrated by comparing the average curvature at a point to its orientation. We construct the average shape of the valley head by averaging the rescaled contours along the arc length. Rewriting equation (5.1) in terms of the width, we obtain

$$
w \kappa=\pi \cos \theta .
$$

Plotting $w \kappa$ as a function of $\cos \theta$, we indeed observe this proportionality (Fig. 4b). Moreover the measured slope $p=3.07 \pm 0.17$ is consistent with the predicted prefactor $p=\pi$. The proportionality relationship holds most clearly at high curvatures, where the approximation that flux scales with curvature is most accurate. Notably, were amphitheater-shaped valley heads semi-circular, then Fig. 4b would show the horizontal line $w \kappa(\theta)=2$. If valley heads were sections of an ellipse with an aspect ratio of $\pi$, the 
Florida
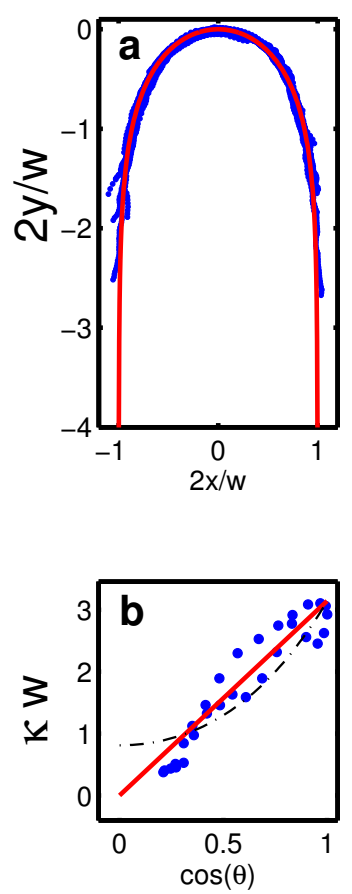

Experiment
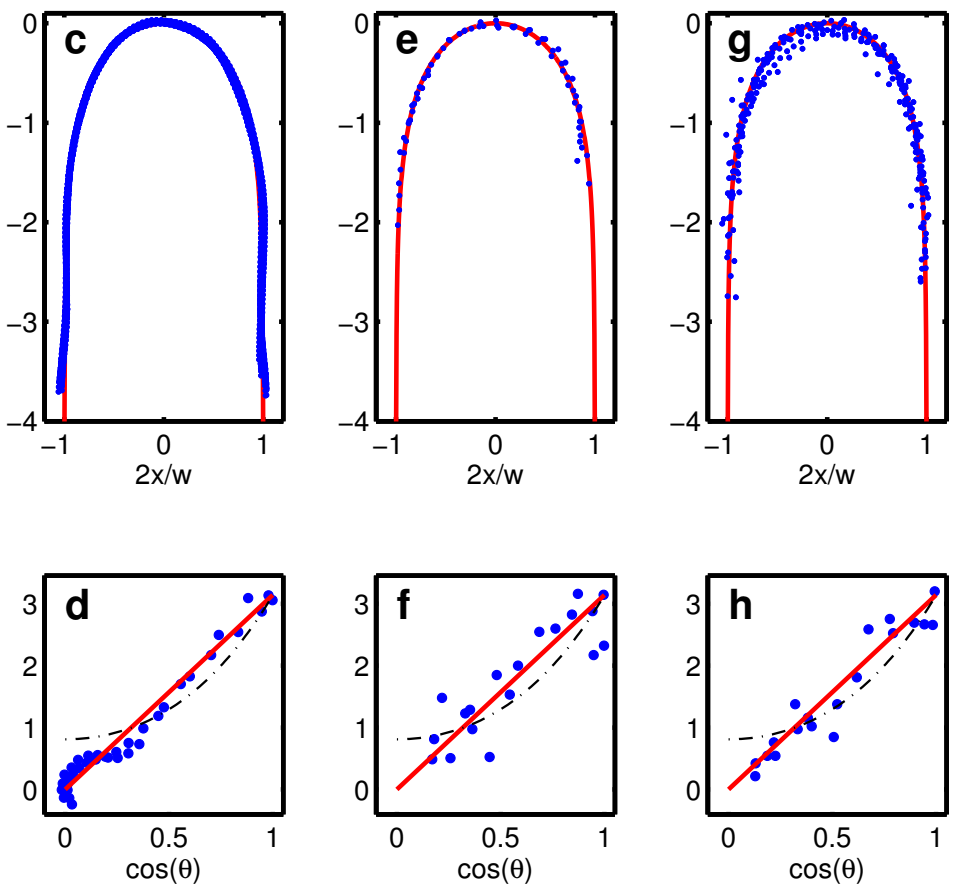

Figure 4. The shape of valley heads in the field, experiments, on Earth, and on Mars are consistent with curvature-driven growth. (a) The shape of a channel produced by curvature-driven growth (red line) compared to the relative positions of points (blue dots) on the edge of valleys from the Florida network (17 elevation contours). (b) Comparison of the curvature at a point to the orientation (blue dots) of valleys from the Florida network. The red line is the linear relationship given in equation (6.1). The black dashed line corresponds to an ellipse with aspect ratio $\pi$. A semi-circular head would predict the horizontal line $\kappa w=2$. (c-d) The analogous plots for the experiments (25 elevation contours extracted at 3 minute intervals). (e-f) The analogous plots for three valleys near Box Canyon and Malad Gorge. $(\mathbf{g}-\mathbf{h})$ The analogous plots for 10 Martian ravines.

data in Fig. 4b would follow the curve $w \kappa(\theta)=\left(4+\left(\pi^{2}-4\right) \cos ^{2} \theta\right)^{3 / 2} / \pi^{2}$. Viewing the semi-circle and ellipse as geometric null hypotheses, we conclude from visual inspection of Fig. 4b that we can confidently reject them in favor of equation (5.2).

Seepage channels can also be grown in the laboratory by forcing water through a sand pile (Schorghofer et al. 2004; Howard 1988; Lobkovsky et al. 2007). Because these channels grow on the time scale of minutes to hours, one can directly observe the development of the channels. Fig. 4c compares equation (5.2) to elevation contours extracted from a previous experiment (Lobkovsky et al. 2007) while the channel is growing. Once the contours are rescaled and averaged, the curvature again is proportional to $\cos \theta$ (Fig. 4d). The measured proportionality constant $p=3.07 \pm 0.13$, consistent with $p=\pi$.

\section{Generalizations}

The strong correspondence between equation (6.1) and the observed shapes of valley heads suggests that amphitheater-shaped heads take their form from curvature-driven growth. Because curvature-driven growth is a simple geometric growth model, it likely 
characterizes a class of physical processes (Brower et al. 1983). For example, when a low-viscosity fluid is pushed slowly into a viscous fluid in two-dimensions, the diffusing pressure field deforms the intruding fluid into an elongated form as described by the Saffman-Taylor instability (Saffman \& Taylor 1958). When stabilized by surface tension, the shape of the resulting "viscous finger" is exactly that given in equation (5.2) (Bensimon et al. 1986; Combescot et al. 1986). This morphology has also been related to the shape of dendrites (Mullins \& Sekerka 1963; Kessler et al. 1986) and is a steady state solution to the deterministic Kardar-Parasi-Zhang equation (Kardar et al. 1986).

This generality leads us to conjecture that when the growth of a valley head responds linearly to a diffusive flux, its dynamics at equilibrium reduce to curvature-driven growth. Geophysically relevant processes in which the growth may be dominated by a (possibly non-linear) diffusive flux include the conduction of heat, topographic diffusion (Culling 1960), the shallow water equations (Chanson 1999), and elastic deformation (Landau \& Lifshitz 1995). Thus, assuming appropriate boundary conditions exist, processes such as seasonal thawing, the relaxation of topography, overland flow, and frost heave may produce valleys indistinguishable in planform shape from seepage channels.

To confirm the wide applicability of he geometric growth model, we proceed to compare equations (5.2) and (6.1) to enigmatic valleys on Earth and Mars. The origins of amphitheater-shaped heads from the Snake River in Idaho (Russell 1902; Lamb et al. 2008) and the Martian valleys of Valles Marineris have been the subject of much debate (Higgins 1982; Malin \& Carr 1999; Sharp \& Malin 1975; Lamb et al. 2006). Fig. 4(eh) shows that the shape of valley heads in both of these systems is consistent with equations (5.2) and (6.1). Averaging the rescaled valleys and comparing the dimensionless curvature to the orientation, we find $p=2.92 \pm 0.24$ and $p=3.02 \pm 0.21$ for the Snake River and Martian features respectively. Both estimates are consistent with $p=\pi$.

\section{Conclusion}

That these valleys are consistent with the predictions of curvature-driven growth does not, however, necessarily imply that their growth was seepage-driven. We favor instead a more conservative conclusion: diffusive transport is ubiquitous and therefore so too is the $\log \cos \theta$ form.

Our results clarify the debate about the origin of amphitheater-shaped valley heads by placing them within a class of dynamical phenomena characterized by growth proportional to curvature. From this qualitative distinction we obtain a quantitative prediction: the valley head has a precisely defined shape with an aspect ratio of $\pi$. Regardless of the specific mechanical processes that cause a particular valley head to grow, all valley heads that fall within this dynamical class will look alike.

We would like to thank The Nature Conservancy for access to the Apalachicola Bluffs and Ravines Preserve, and K. Flournoy, B. Kreiter, S. Herrington and D. Printiss for guidance on the Preserve. We thank B. Smith for her experimental work. It is also our pleasure to thank M. Berhanu. This work was supported by Department of Energy Grant FG02-99ER15004. O. D. was additionally supported by the French Academy of Sciences.

\section{REFERENCES}

Abrams, D M., Lobkovsky, A E, Petroff, A P, Straub, K M, McElroy, B., Mohrig, D C, Kudrolli, A. \& Rothman, D H 2009 Growth laws for channel networks incised by groundwater flow. Nature Geoscience 28 (4), 193-196. 
BeAR, J. 1979 Hydraulics of groundwater. McGraw-Hill New York.

Ben-Jacob, E., Goldenfeld, N., Langer, JS \& Schön, G. 1983 Dynamics of interfacial pattern formation. Physical Review Letters 51 (21), 1930-1932.

Bensimon, D., Kadanoff, L P, Liang, S., Shraiman, B I \& Tang, C. 1986 Viscous flows in two dimensions. Reviews of Modern Physics 58 (4), 977-999.

Brower, R C, Kessler, D A, Koplik, J. \& Levine, H. 1983 Geometrical approach to moving-interface dynamics. Physical Review Letters 51 (13), 1111-1114.

Chanson, H. 1999 The hydraulics of open channel flow: an introduction. Arnold London.

Combescot, R., Dombre, T., Hakim, V., Pomeau, Y. \& Pumir, A. 1986 Shape selection of Saffman-Taylor fingers. Physical Review Letters 56 (19), 2036-2039.

Culling, W E H 1960 Analytic theory of erosion. J. Geol. 68, 336-344.

Dunne, T. 1980 Formation and controls of channel networks. Progress in Physical Geography $4(2), 211$.

HigGins, C G 1982 Drainage systems developed by sapping on Earth and Mars. Geology 10 (3), 147.

HowARD, A D 1988 Groundwater sapping experiments and modeling. Sapping Features of the Colorado Plateau: A Comparative Planetary Geology Field Guide pp. 71-83.

Kardar, M., Parisi, G. \& Zhang, Y.C. 1986 Dynamic scaling of growing interfaces. Physical Review Letters 56 (9), 889-892.

Kessler, D.A., Koplik, J. \& Levine, H. 1985 Geometrical models of interface evolution. III. Theory of dendritic growth. Physical Review A 31 (3), 1712-1717.

Kessler, D.A., Koplik, J. \& Levine, H. 1986 Dendritic growth in a channel. Physical Review A 34 (6), 4980-4987.

LAity, J E \& Malin, M C 1985 Sapping processes and the development of theater-headed valley networks on the Colorado Plateau. Geological Society of America Bulletin 96 (2), 203.

Lamb, M P, Dietrich, W E, Aciego, S M, DePaolo, D J \& Manga, M. 2008 Formation of Box Canyon, Idaho, by megaflood: Implications for seepage erosion on Earth and Mars. Science 320 (5879), 1067.

Lamb, M P, Howard, A D, Johnson, J., Whipple, K X, Dietrich, W E \& Perron, J T 2006 Can springs cut canyons into rock. J. Geophys. Res 111.

Landau, L D \& Lifshitz, E M 1995 Theory of Elasticity, Course of Theoretical Physics vol 7. VII, 3rd revised edition (Butterworth-Heinemann, London).

Lobkovsky, A E, Smith, B E, Kudrolli, A., Mohrig, D C \& Rothman, D H 2007 Erosive dynamics of channels incised by subsurface water flow. J. Geophys. Res 112.

Malin, M C \& CARR, M H 1999 Groundwater formation of Martian valleys. Nature 397 (6720), $589-591$.

Marsili, M., Maritan, A., Toigo, F. \& Banavar, J.R. 1996 Stochastic growth equations and reparametrization invariance. Reviews of Modern Physics 68 (4), 963-983.

Mullins, WW \& SEKERKA, RF 1963 Morphological stability of a particle growing by diffusion or heat flow. Journal of Applied Physics 34, 323.

Orange, D L, Anderson, R S \& Breen, N A 1994 Regular canyon spacing in the submarine environment: the link between hydrology and geomorphology. GSA Today 4 (2), 35-39.

PELCÉ, P. 1988 Dynamics of curved fronts. Academic Pr.

PelCÉ, P. 2004 New visions on form and growth: fingered growth, dendrites, and flames. Oxford University Press, USA.

Russell, I C 1902 Geology and water resources of the Snake River Plains of Idaho. US Geol Survey Bull 199, 1-192.

SAFFMAN, P G \& TAYLOR, G I 1958 The penetration of a fluid into a porous medium or HeleShaw cell containing a more viscous liquid. Proceedings of the Royal Society of London. Series A, Mathematical and Physical Sciences 245 (1242), 312-329.

Schorghofer, N., Jensen, B., Kudrolli, A. \& Rothman, D H 2004 Spontaneous channelization in permeable ground: theory, experiment, and observation. Journal of Fluid Mechanics 503, 357-374.

Schumm, S A, Boyd, K F, Wolff, C G \& Spitz, WJ 1995 A ground-water sapping landscape in the Florida Panhandle. Geomorphology 12 (4), 281-297. 
Sharp, R P \& Malin, M C 1975 Channels on Mars. Geological Society of America Bulletin 86 (5), 593.

Shraiman, B. \& Bensimon, D. 1984 Singularities in nonlocal interface dynamics. Physical Review A 30 (5), 2840-2842.

Wentworth, C K 1928 Principles of stream erosion in Hawaii. The Journal of Geology 36 (5), 385-410. 


\title{
Supplementary Material
}

\section{for \\ Geometry of Valley Growth}

\begin{abstract}
Alexander P. Petroff ${ }^{1}$, Olivier Devauchelle ${ }^{1}$, Daniel M. Abrams ${ }^{1,2}$,
\end{abstract} Alexander E. Lobkovsky ${ }^{1}$, Arshad Kudrolli ${ }^{3}$, Daniel H. Rothman ${ }^{1}$

${ }^{1}$ Department of Earth, Atmospheric and Planetary Sciences, Massachusetts Institute of Technology, Cambridge, MA 02139 USA

${ }^{2}$ Present address: Department of Engineering Sciences and Applied Mathematics, Northwestern University, Evanston, IL 60208

${ }^{3}$ Department of Physics, Clark University, Worcester, MA 01610

\section{Contents}

1 Computation of the water table $\quad$ S2

2 Selection of the boundary $\quad$ S2

3 Comparison of the shape of the water table to the Poisson elevation S2

4 Comparison of contour curvature to the groundwater flux S5

5 The Poisson flux-curvature relation $\quad$ S5

$\begin{array}{lll}6 & \text { Derivation of the shape of the valley head } & \text { S7 }\end{array}$

7 Selection of valley heads $\quad$ S8

7.1 Florida Network . . . . . . . . . . . . . . . . . . . . . S9

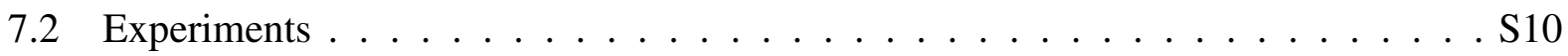

7.3 Snake River valley heads . . . . . . . . . . . . . . . . . . S11

7.4 Martian valley heads . . . . . . . . . . . . . . . . S12

8 Stream discharge data $\quad$ S13

8.1 Comparison of field measurements to the predicted flux . . . . . . . . . S13

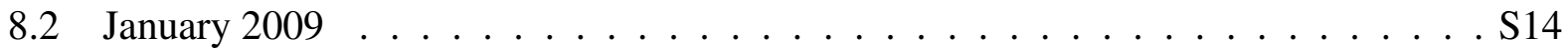

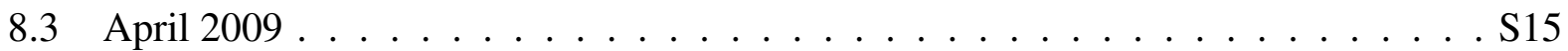




\section{Computation of the water table}

In order to find the distribution of groundwater flux into the network, we solved for the shape of the water table around the channels. From the main text, the Poisson elevation $\phi$ of the water table is a solution to the equation:

$$
\nabla^{2} \phi^{2}+1=0
$$

with absorbing and zero flux boundary conditions. Thus $\phi$ is independent of the hydraulic conductivity $K$.

The ground water flux at a point is related to the shape of the watertable through the equation

$$
q=\frac{K}{2}\left\|\nabla h^{2}\right\|
$$

from which

$$
q=P\left\|\nabla \frac{K}{2 P} h^{2}\right\|
$$

Thus, from the definition of $\phi$

$$
q=P\left\|\nabla \phi^{2}\right\|
$$

Because $\phi$ is only a function of the network geometry, $q$ is independent of $K$. This result also follows from conservation of mass. The total discharge from the network must be equal to the total rain that falls into the network, regardless of conductivity. $K$ sets the slope of watertable at the boundary required to maintain this flux.

\section{Selection of the boundary}

We solve the equation around a boundary chosen to follow the position of springs and streams. To identify such a boundary, we first remove the mean slope (0.0025) of the topography. We then chose the $45 \mathrm{~m}$ elevation contour of the resulting topography as the boundary (Figure S1) obtained from a high resolution LIDAR map of the network (S1). This elevation was chosen as the approximate elevation of many springs. When the contour exits the area where the LIDAR map was available, we replace the missing section of the channel with an absorbing boundary condition. Because this approximation results in uncertainties in the flux near the missing boundary, we only analyze the water flux into a well contained section of the network (blue boundary in Figure S1). Finally, we include a zero-flux boundary condition in the south east in the approximate location of a drainage divide. We solve equation (S1) with these boundary conditions using a finite-element method $(S 2)$.

\section{Comparison of the shape of the water table to the Poisson elevation}

Here we show that the solution of equation (S1) is consistent with field observations. We compare $\phi$ (Figure S2b) to a previously reported (S1) ground penetrating radar (GPR) survey of the channels (Figure S2c). 


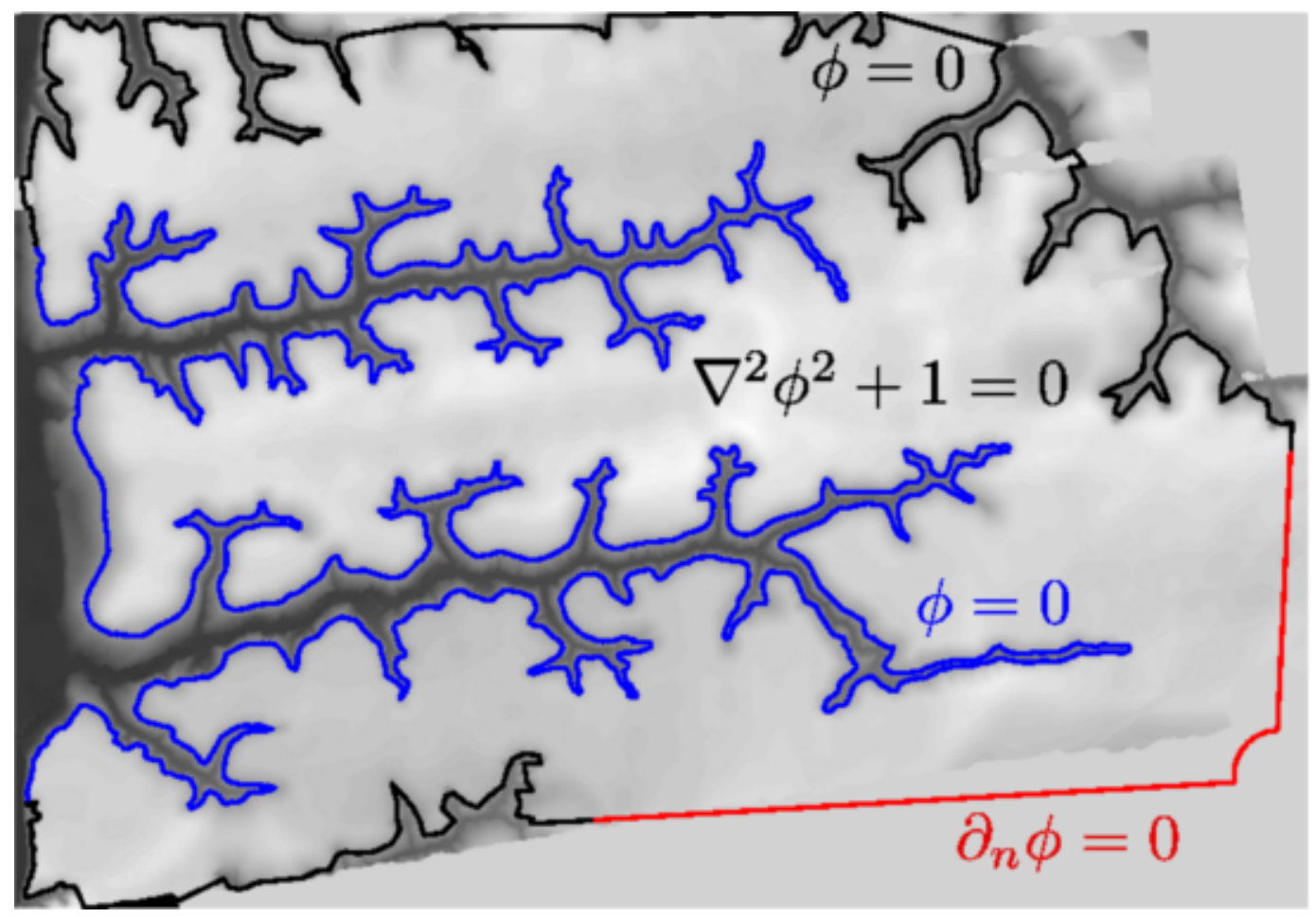

Figure S1: To most closely approximate the shape of the network we use an elevation contour of the topography. Approximating the channels as nearly flat, we required that the water table intersect the channels at a constant height, which we chose as zero. This boundary is drawn in blue and black. Additionally, a drainage divide (red line) was included in the south east. Because our LIDAR map (S1) only shows two full valley networks, we only analyze the data from this portion of the boundary (blue line). The boundary is linearly interpolated between points spaced at 20 $\mathrm{m}$ intervals on the blue boundary and points spaced by an average of $50 \mathrm{~m}$ on the red and black boundaries.

As all heights are measured relative to the impermeable layer, we define $h_{0}$ to be the reference elevation and shift $h$ accordingly. It follows from the definition of $\phi$ that

$$
h=h_{0}+\sqrt{\frac{2 P}{K} \phi^{2}+\left(h_{B}-h_{0}\right)^{2}},
$$

where $h_{B}$ is the elevation of the water table at the boundary. A least squares fit of the measured elevations to equation (S5) gives estimates $P / K=7 \times 10^{-5}, h_{0}=38 \mathrm{~m}$, and $h_{B}=38 \mathrm{~m}$ (figure S2d). Additionally taking $P$ to be the observed mean rainfall rate of $5 \times 10^{-8} \mathrm{~m} \mathrm{sec}^{-1}$, gives $K=6 \times 10^{-4} \mathrm{~m} \mathrm{sec}^{-1}$. Each of these estimates is consistent with the analysis of Ref. ( $\left.S 1\right)$. Furthermore, the estimated permeability is consistent with the permeability of clean sand (S3). The elevation $h_{0}$ of the impermeable layer may be overestimated due to uncertainties in the analysis of the GPR data. 


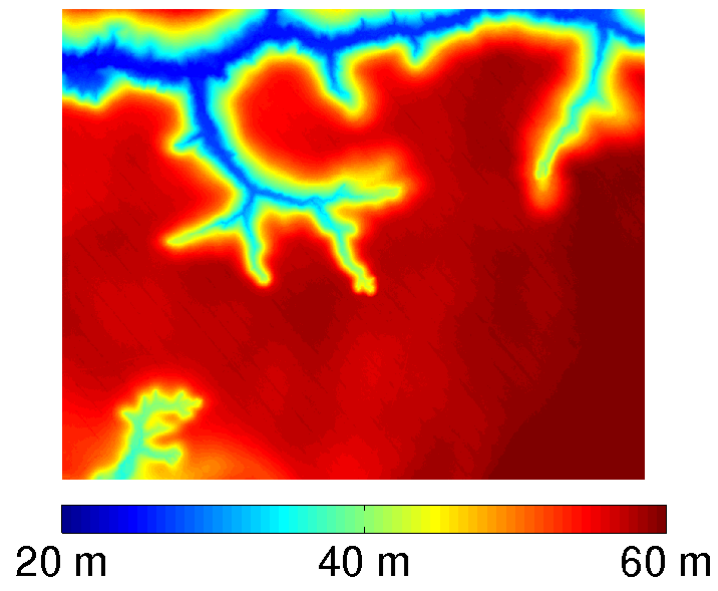

(a)

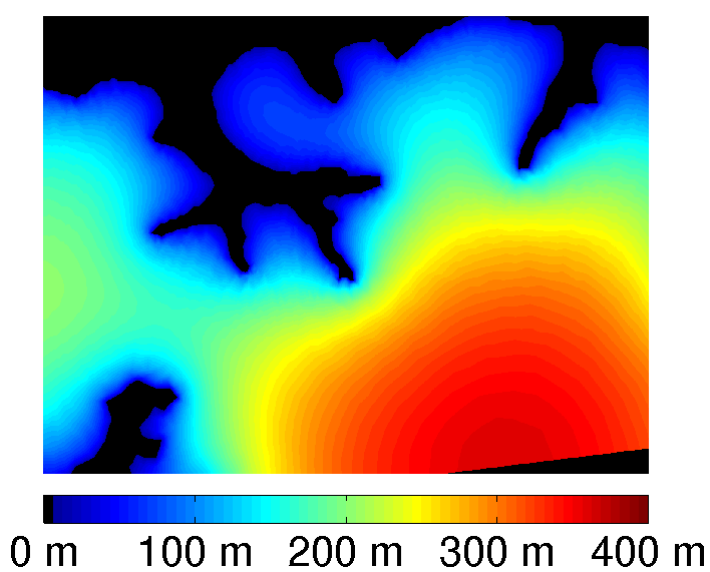

(b)

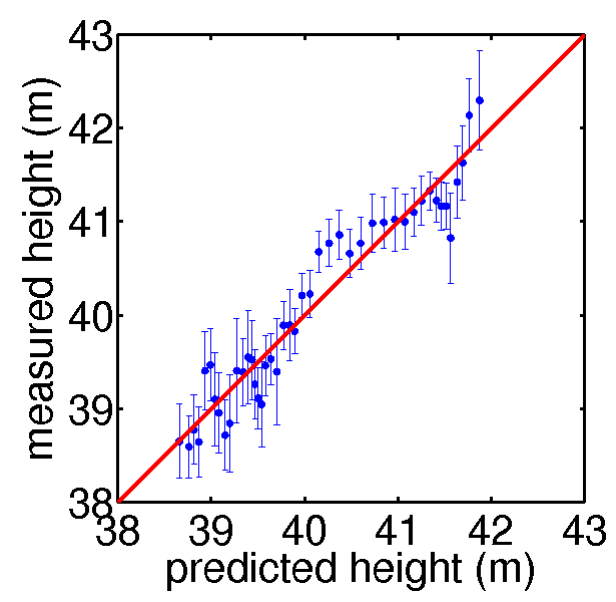

(d)

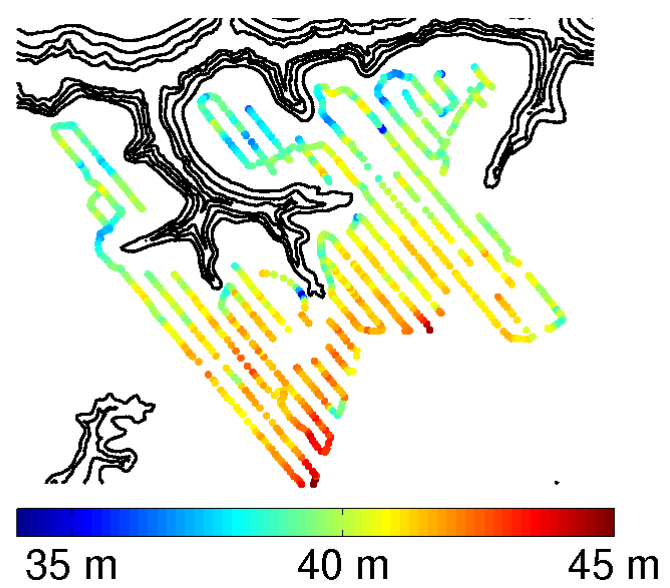

(c)

Figure S2: Comparison of the Poisson elevation to field observation. (a) The available ground penetrating radar survey was conducted on a portion of the southern valley network. The topographic map of the channels near the survey is $1400 \mathrm{~m}$ across. (b) We solved equation (S1) around the valley for the Poisson elevation. (c) The ground penetrating radar survey (S1) provided the elevation of the water table above sea level at 1144 points around the network. The valley walls are represented by the elevation contours for $30 \mathrm{~m}$ to $45 \mathrm{~m}$ at $5 \mathrm{~m}$ intervals. (d) The measured height is consistent with theory. The red line indicates perfect agreement. 


\section{Comparison of contour curvature to the groundwater flux}

Because the curvature is a function of the second derivative of a curve, its estimation requires an accurate characterization of the channel shape. To have the highest possible accuracy in the estimation of curvature, we restrict the comparison between flux and curvature to a small piece of the network where the boundary is linearly interpolated between points separated by $5 \mathrm{~m}$.

The curvature at a point on the boundary is computed by fitting a circle to the point and its neighbors on both sides (Figure S3b). Given the best fitting circle, the magnitude of the curvature is the inverse of the radius. The curvature is negative when the center of the circle is outside the valley and positive when the center is inside the valley.

To compare the curvature and flux at a point, we calculate the Poisson flux $q_{p}$ into each section of this piece of the network by solving equation (S1) between the channels (Figure S3c). We closed the boundary on the eastern side of the domain by attaching the extremities to the valley network to the east using zero-flux boundaries. To identify the characteristic dependence of the flux at a point on the curvature, we averaged the flux and curvature at points on the boundary with similar curvatures. Each point in Figure S3d represents the average flux and curvature of 50 points on the boundary.

\section{The Poisson flux-curvature relation}

The Poisson flux is the area that drains into small segment of the network divided by the length of the segment. It can therefore be considered as a "local" inverse drainage density. Because all of the area drains into some piece of the channel, the integral of the Poisson flux is the total area of the basin. It follows that its mean value is the inverse drainage density.

In what follows we ask how the Poisson flux depends on the distance $d$ a piece of the network is from its drainage divide. We note that if a $d$ has a characteristic value in a network, then we find a scaling of geometric flux with curvature that is consistent with observation (Fig. 1c).

A section of the network receives a large flux when it drains a large area $a$ or when all of the water is forced through a small length of channel wall $\ell$. When water from a large basin $\left(d \gg \kappa^{-1}\right)$ drains toward a point, then $a \sim d^{2}$ (Fig. S4a). Note that " $\sim$ " is the symbol for "is the order of magnitude of" or "scales as." This area is drained into a section of channel, the length $\ell$ of which is proportional to the planform radius of curvature, $\kappa^{-1}$; thus in regions of high curvature

$$
q_{p}=\Omega \kappa,
$$

where $\Omega=m d^{2}$ is a constant of the network related to the characteristic groundwater discharge of a head and $m \sim 1$ is a proportionality constant related to the characteristic shape of a valley head. The flux into a point is therefore proportional to the product of variables characterizing the network, $\Omega$, and the local geometry of the channel, $\kappa$. Equating $d$ with the inverse drainage density of the network, we find $d=147 \mathrm{~m}$ from the analysis of the topographic map. Fitting a hyperbola to the data in Fig. 1c, given this value of $d$, gives $m=1.5 \pm 0.2$, consistent with $m \sim 1$.

In concave regions of the channel the area drained is the sum of the area outside the concavity and the area inside the concavity (Fig. S4b). This area $a$ can be expressed as

$$
a=m_{1} d \kappa^{-1}+m_{2} \kappa^{-2},
$$




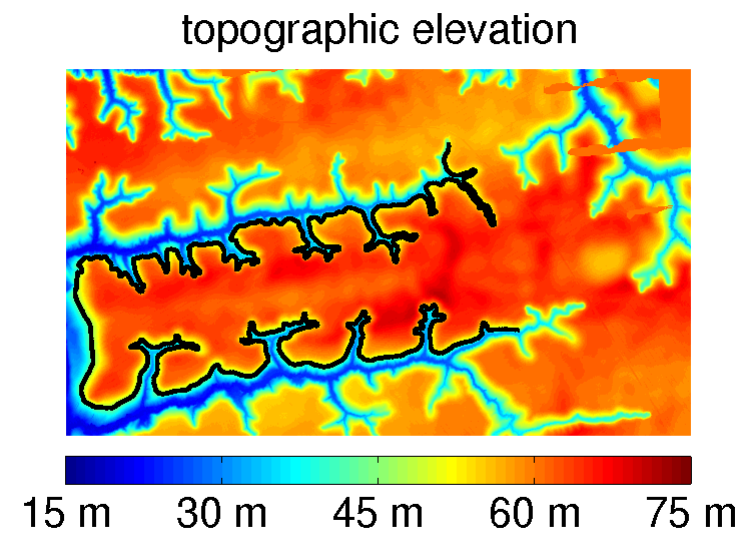

(a)

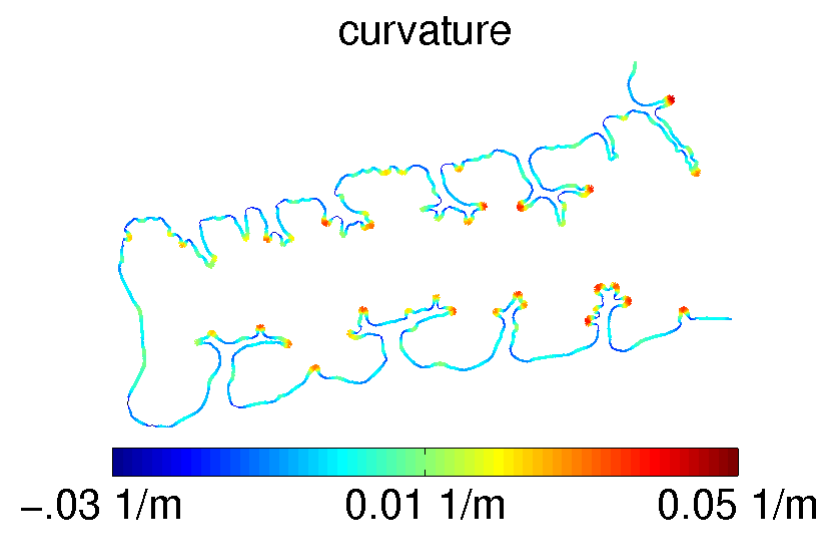

(b)

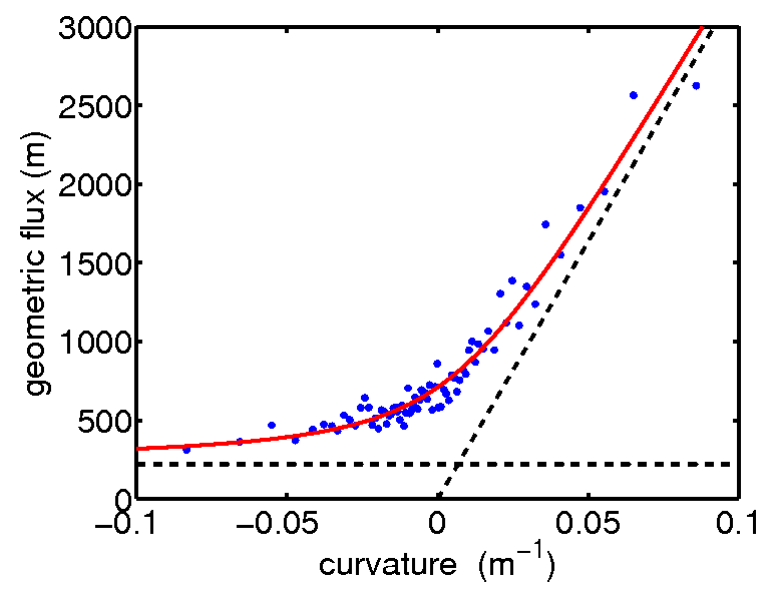

(d)

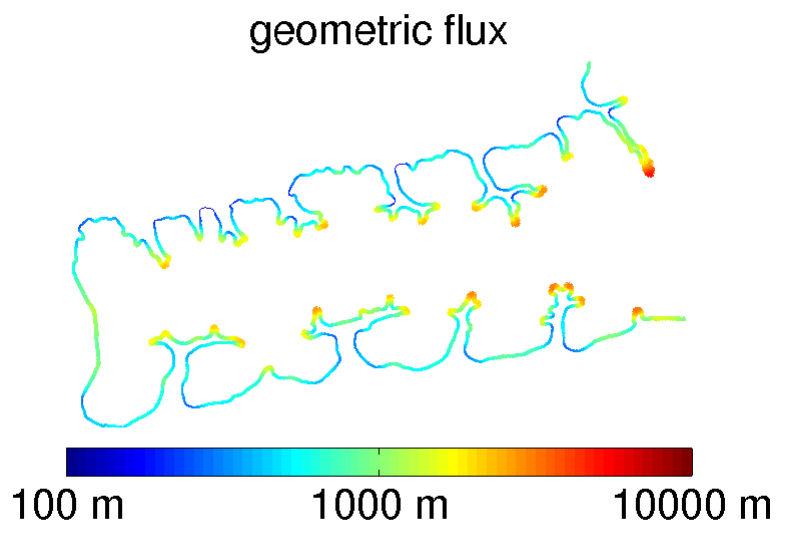

(c)

Figure S3: Identification of the relationship between the curvature of the valley walls and the local flux of groundwater. (a) The curvature and flux are measured between two valleys along the black contour. (b) The curvature at each point on the boundary is measured by fitting a circle to boundary. (c) The flux into each section of the network is found from the solution of equation (S1). (d) Comparison of the flux into each section of the network to the curvature. Geometric reasoning gives the asymptotic behavior (black dashed lines) of this relation when the magnitude of the curvature is large. 

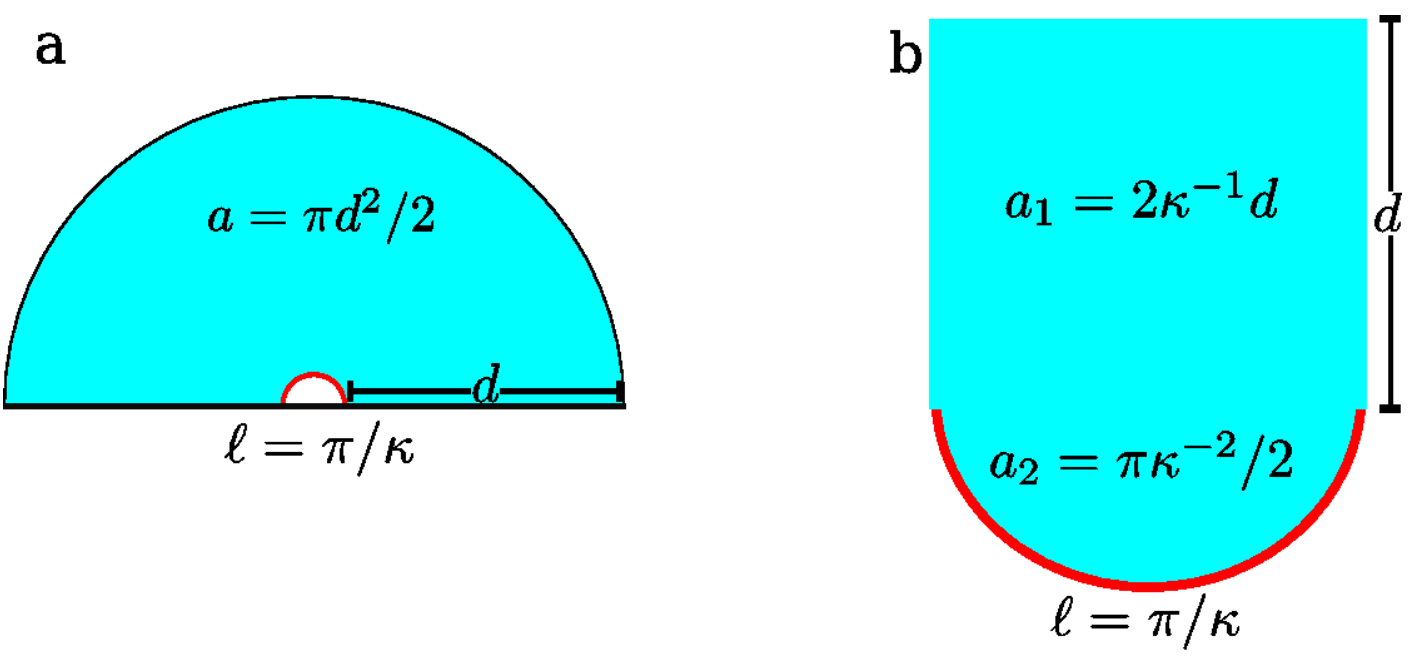

Figure S4: The Poisson flux is the local drainage density. (a) When a basin drains into a convex region (red line) the drainage density increases with curvature $\kappa$. (b) When a basin drains into a concave interval, the drainage density decreases with curvature.

where $m_{1}$ and $m_{2}$ are dimensionless numbers related to the shape of the drainage basin outside and inside the concavity, respectively. For example, if the concavity is a semi-circular depression and it drains a rectangular region, then $m_{1}=2$ and $m_{2}=\pi / 2$. This area is drained by a segment of length $\sim \kappa^{-1}$ giving a mean Poisson flux $q_{p}$ that scales as

$$
q_{p}=\left(m_{1} d+m_{2} \kappa^{-1}\right) / m_{3},
$$

where $m_{3}$ is a dimensionless number related to the shape of the concavity. Fitting the data to a hyperbola, and again taking $d=147 \mathrm{~m}$, we find $m_{1} / m_{3}=1.52 \pm 0.22$ and $m_{2} / m_{3}=10.80 \pm 2.97$. This scaling relation, in combination with the behavior at large positive $\kappa$, gives the the asymptotic behavior of the flux-curvature relation.

\section{Derivation of the shape of the valley head}

Here we derive equation (5) of the main text.

The balance between translation and curvature-driven growth relates the orientation to the curvature through the equation

$$
\pi \cos \theta=w \kappa .
$$

We first re-write the orientation of a segment in terms of the local normal $\hat{n}(x)$ to the curve and the direction the head is translating $\hat{y}$. It follows from the definition of $\theta$ that

$$
\pi \hat{n}(x) \cdot \hat{y}=w \kappa(x)
$$

Next, by describing the shape of a valley head by a curve $y(x)$, equation (S10) becomes

$$
\frac{-\pi}{\sqrt{1+\left(\partial_{x} y\right)^{2}}}=w \frac{\partial_{x x} y}{\left(1+\left(\partial_{x} y\right)^{2}\right)^{3 / 2}} \text {. }
$$


With the substitution $g=\partial_{x} y$, this equation is re-expressed as an integrable, first order equation as

$$
w \partial_{x} g+\pi\left(1+g^{2}\right)=0 .
$$

Integrating once,

$$
g=\partial_{x} y=-\tan \left(\frac{\pi x}{w}\right)
$$

Integrating a second time for $y$ gives

$$
y=\frac{w}{\pi} \log \cos \left(\frac{\pi x}{w}\right),
$$

equivalent to equation (5) of the main text.

Although not necessary here, it is occasionally useful to express the shape of the channel as a vector $\mathbf{v}$ parameterized by arc length $s$,

$$
\mathbf{v}(s)=\frac{w}{\pi}\left(\begin{array}{c}
2 \arctan (\tanh (\pi s / 2 w)) \\
\log (\operatorname{sech}(\pi s / w))
\end{array}\right) .
$$

The derivative $\mathbf{v}$ is the unit tangent vector.

\section{Selection of valley heads}

The derivation of equation (S14) requires that the channel grow forward without changing shape. Consequently, when identifying seepage valley heads suitable for analysis, we restricted our analysis to isolated channels.

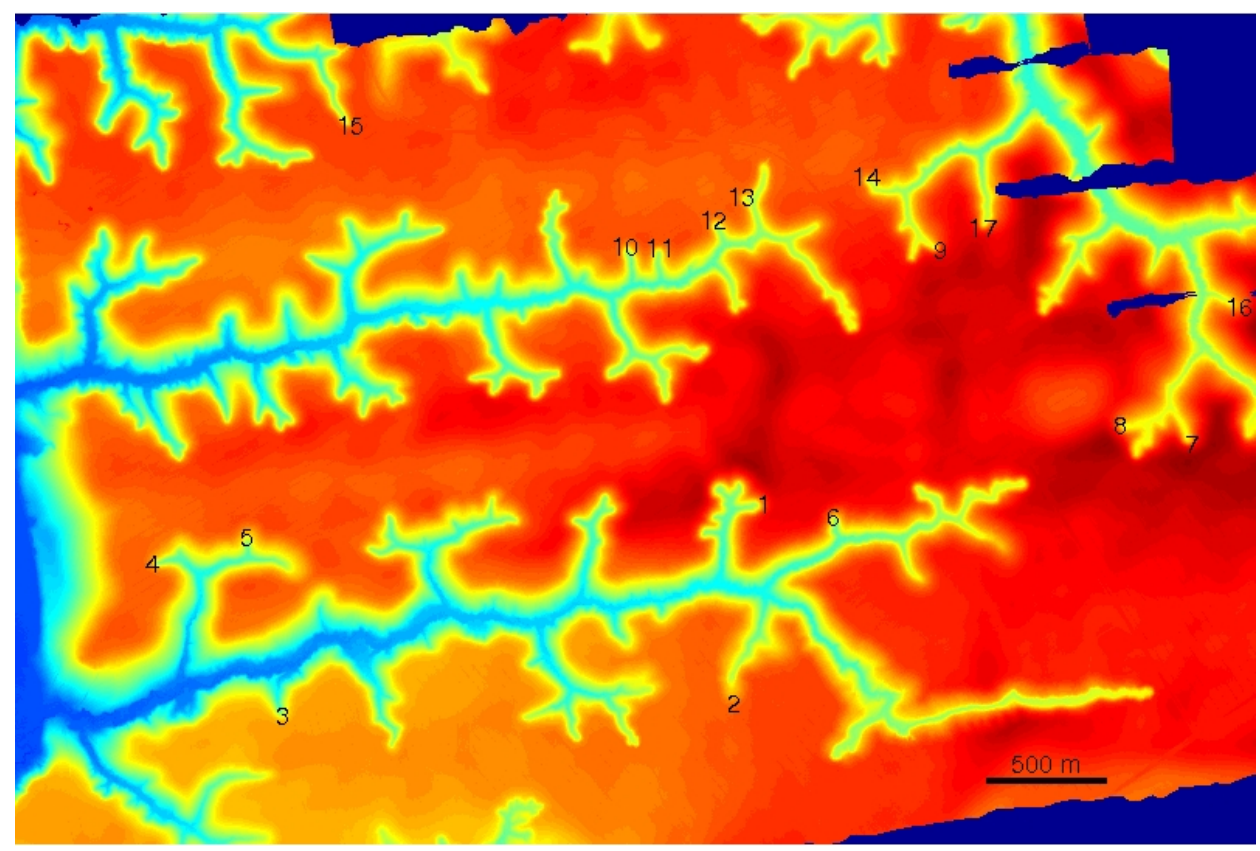

Figure S5: 17 isolated valley heads were chosen from the Florida network 


\subsection{Florida Network}

We select valley heads from the Florida network that are reasonably isolated and not bifurcating. Given such a valley, we extract an elevation contour approximately one half the distance between the spring and the upland flat plain. We find that the deviation in the shape of any given channel from equation (S14) is insensitive to the choice of elevation contour.

Table S1: Valley heads from the Florida network. Coordinates are given with respect to UTM zone $16 \mathrm{R}$

\begin{tabular}{ccccc} 
channel & Easting $(\mathrm{m})$ & Northing $(\mathrm{m})$ & elevation $(\mathrm{m})$ & width $(\mathrm{m})$ \\
\hline \hline 1 & 696551.40 & 3373949.52 & 56.91 & 100.66 \\
2 & 696423.49 & 3373123.32 & 55.74 & 111.03 \\
3 & 694537.55 & 3373068.53 & 42.93 & 50.47 \\
4 & 693995.09 & 3373701.11 & 49.49 & 49.60 \\
5 & 694391.80 & 3373813.01 & 43.49 & 38.72 \\
6 & 696841.09 & 3373900.80 & 44.23 & 28.96 \\
7 & 698339.72 & 3374200.55 & 59.95 & 83.53 \\
8 & 698040.54 & 3374282.69 & 50.91 & 48.28 \\
9 & 697285.68 & 3375011.47 & 59.12 & 80.20 \\
10 & 695968.97 & 3375029.24 & 49.15 & 56.05 \\
11 & 696114.42 & 3375019.47 & 46.62 & 42.59 \\
12 & 696336.97 & 3375135.23 & 49.88 & 56.34 \\
13 & 696453.90 & 3375233.09 & 51.13 & 50.54 \\
14 & 696976.13 & 3375317.38 & 51.10 & 46.08 \\
15 & 694818.57 & 3375532.39 & 54.82 & 52.04 \\
16 & 698537.91 & 3374777.58 & 54.21 & 70.77 \\
17 & 697463.52 & 3375108.63 & 53.97 & 55.17
\end{tabular}




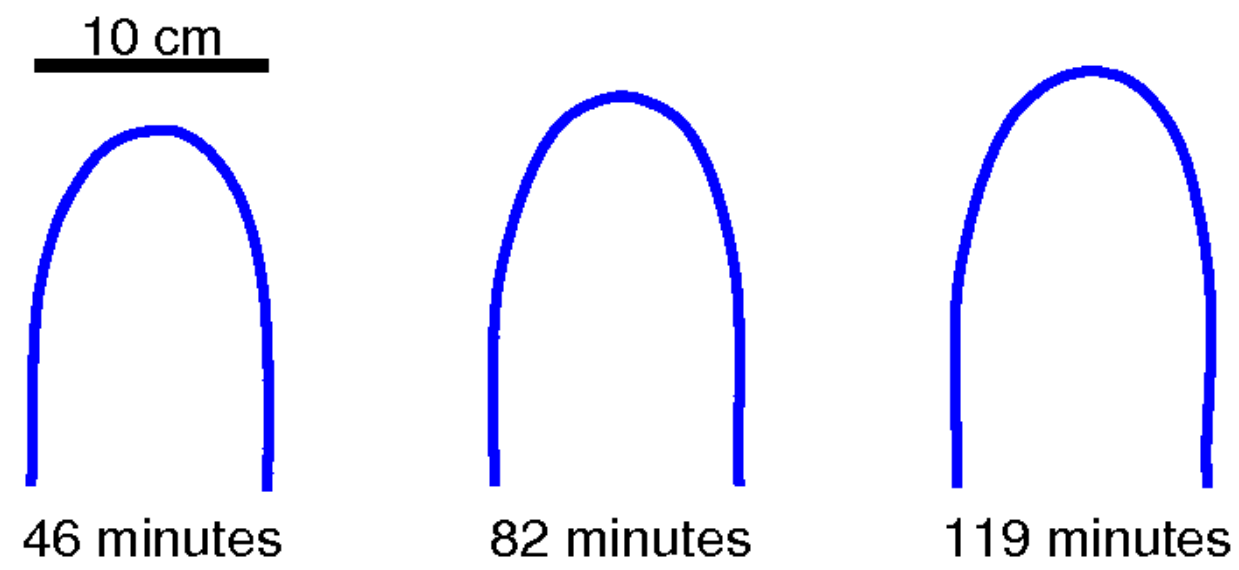

Figure S6: An elevation contour (blue lines) was extracted from the experiment every three minutes from a digital elevation map (S5). These three representative elevation contours from the beginning, middle, and end of the experiment demonstrate that the shape changed little during growth.

\subsection{Experiments}

The experimental apparatus used to grow seepage channels has been previously described $(S 4)$. The channel used in the comparison to equation (S14) grew from an initially rectangular indentation $3 \mathrm{~cm}$ deep in a bed of $0.5 \mathrm{~mm}$ glass beads sloped at an angle of $7.8^{\circ}$ with a pressure head of $19.6 \mathrm{~cm}$. To extract the shape of the channel, we first removed the slope of the bed by subtracting the elevation of each point at the beginning of the experiment. We then follow the growth of an elevation contour a constant depth below the surface. Because the shape of channel at the beginning of the experiment is heavily influenced by the shape of the initial indentation, we restrict our analysis to the shape of the contour after 45 minutes of growth. The channel grew for a total of 119 minutes and was measured at 3 minute intervals. 


\subsection{Snake River valley heads}

To compare the form of amphitheater-shaped valley heads growing off of the Snake River in Idaho, we extract the valley shape from images taken from Google Earth. We select three prominent heads (Table S2, Figure S7); Box Canyon (S6) and two near Malad Gorge. We extract the shape of each of these heads by selecting points at the upper edge of the valley head. The mean spacing between points is $13 \mathrm{~m}$. We stop selecting points when the valley turns away from the head.

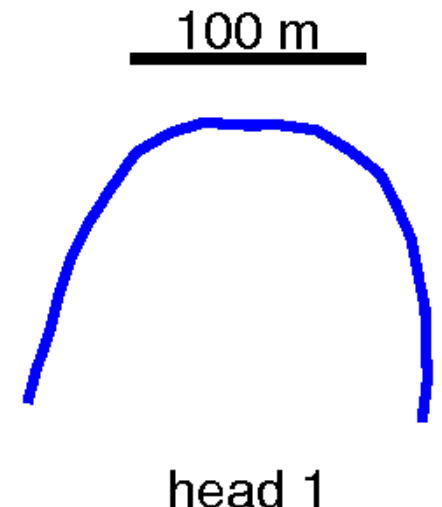

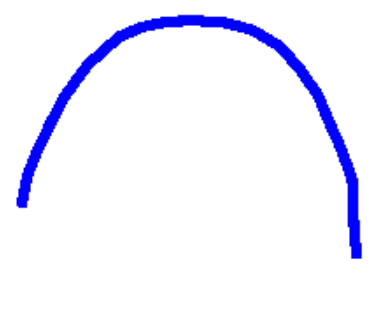

head 2

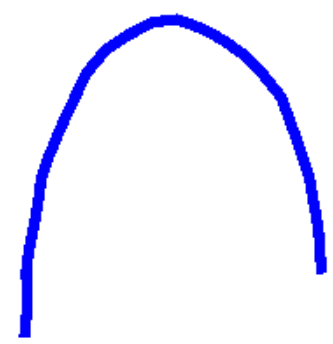

head 3

Figure S7: The shape of amphitheater-shaped valley heads growing off of the Snake River in Idaho were extracted from aerial photos of the channels. Heads 1 and 2 are near Malad Gorge. Head 3 is Box Canyon.

Table S2: Valley heads near the Snake River

\begin{tabular}{cccc} 
channel & latitude & longitude & width $(\mathrm{m})$ \\
\hline \hline 1 & $42.8675^{\circ}$ & $115.6432^{\circ}$ & 190 \\
2 & $42.8544^{\circ}$ & $115.7045^{\circ}$ & 166 \\
3 & $42.7084^{\circ}$ & $114.9683^{\circ}$ & 132
\end{tabular}




\subsection{Martian valley heads}

The shapes of the Martian ravines which we compared to equation (S14) were extracted from images generated by the Themis camera on the Mars Odyssey orbiter. Channels are selected based on the condition that the amphitheater head was largely isolated from neighboring structures. Because the ravines are deeply incised into the topography, there is typically a sharp contrast between the ravines and the surrounding topography. We extract the shape of the ravine by selecting points spaced of order $100 \mathrm{~m}$ apart along the edge of the ravine (Table S3, Figure S8). We stop selecting points when the ravine intersects with a neighboring structure or when the direction of the valley curves away from the head.

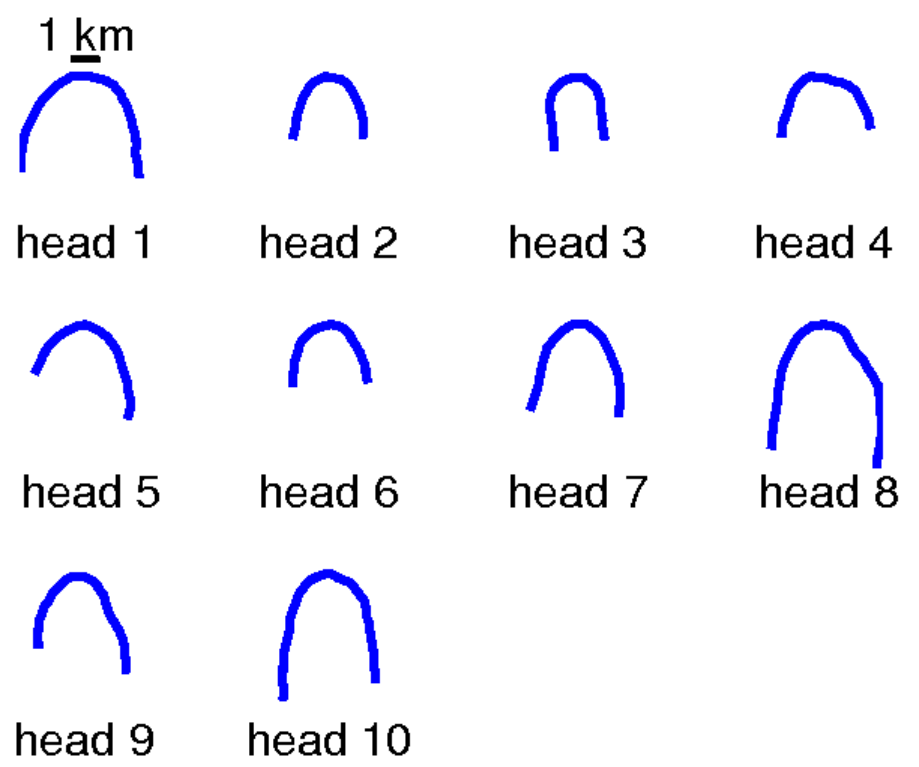

Figure S8: 10 valley heads near the Nirgal Valley, Mars. The shape of each head was extracted by selecting points at the edge of the valley head from images generated by the Mars Odyssey orbiter.

Table S3: Martian valley heads

\begin{tabular}{ccccc} 
head & Themis Image & latitude & longitude & width $(\mathrm{m})$ \\
\hline \hline 1 & V06395001 & $-8.7270^{\circ}$ & $278.1572^{\circ}$ & 4730 \\
2 & V06395001 & $-8.7235^{\circ}$ & $278.1557^{\circ}$ & 2650 \\
3 & V09004001 & $-9.4310^{\circ}$ & $274.6110^{\circ}$ & 1940 \\
4 & V11138002 & $-7.9183^{\circ}$ & $275.4740^{\circ}$ & 3690 \\
5 & V11138002 & $-7.9160^{\circ}$ & $275.4736^{\circ}$ & 3740 \\
6 & V14133002 & $-9.5763^{\circ}$ & $278.4435^{\circ}$ & 2940 \\
7 & V14857001 & $-7.5656^{\circ}$ & $273.6060^{\circ}$ & 3110 \\
8 & V16654002 & $-8.7792^{\circ}$ & $275.5868^{\circ}$ & 3970 \\
9 & V16654002 & $-8.7781^{\circ}$ & $275.5894^{\circ}$ & 3310 \\
10 & V26750003 & $-8.0633^{\circ}$ & $274.8977^{\circ}$ & 3370
\end{tabular}




\section{Stream discharge data}

\subsection{Comparison of field measurements to the predicted flux}

Fig. 1 of the main text compares the solution of equation (S1) to field measurements. The instantaneous discharge of a stream is measured from the the cross-sectional area $a$ in a locally straight section of the channel and the surface velocity $v$, from which the discharge $Q=a v$. We measure the surface velocity of the stream from the travel time of a small passive tracer between points at a fixed distance. This method may underestimate the discharge in very small streams where a substantial fraction of the flow may be moving through the muddy banks of the stream.

To compare the measured discharge to the Poisson equation, we integrate the flux, $q=P\left\|\nabla \phi^{2}\right\|$, along the section of the network upstream from the measurement assuming the reported annual rainfall, $P=5 \times 10^{-8} \mathrm{~m} \mathrm{sec}^{-1}$. When discharge is measured near a spring, the flux is integrated around the valley head. 


\subsection{January 2009}

\begin{tabular}{|c|c|c|c|}
\hline Easting $(\mathrm{m})$ & Northing (m) & discharge $\left(\mathrm{cm}^{3} \mathrm{sec}^{-1}\right)$ & predicted discharge $\left(\mathrm{cm}^{3} \mathrm{sec}^{-1}\right)$ \\
\hline 696905.45 & 3374708.15 & 11700 & 4802.54 \\
\hline 695425.47 & 3374595.35 & 310 & 422.30 \\
\hline 695333.80 & 3374486.16 & 1900 & 1459.50 \\
\hline 695410.03 & 3374422.51 & 2100 & 1403.02 \\
\hline 695589.53 & 3374413.20 & 2000 & 1272.90 \\
\hline 695608.45 & 3374439.65 & 1700 & 1145.17 \\
\hline 695602.26 & 3374467.29 & 4700 & 2103.67 \\
\hline 695532.14 & 3374764.77 & 310 & 490.24 \\
\hline 694045.68 & 3373713.71 & 710 & 1708.72 \\
\hline 694102.24 & 3373742.47 & 850 & 1381.31 \\
\hline 694110.98 & 3373726.59 & 850 & 3520.83 \\
\hline 694393.38 & 3373788.44 & 810 & 1761.83 \\
\hline 694515.20 & 3373714.30 & 2300 & 2051.40 \\
\hline 694700.99 & 3373494.69 & 2900 & 1831.06 \\
\hline 697174.63 & 3373662.18 & 700 & 3004.36 \\
\hline 697622.18 & 3374045.11 & 10800 & 4700.85 \\
\hline 697523.57 & 3374034.52 & 440 & 2225.53 \\
\hline 696432.08 & 3373937.74 & 3500 & 2619.48 \\
\hline 696353.61 & 3374006.59 & 3500 & 2688.01 \\
\hline 696415.16 & 3373979.53 & 3600 & 2545.77 \\
\hline 696363.79 & 3373884.98 & 570 & 673.20 \\
\hline 696314.56 & 3373838.46 & 3100 & 1132.34 \\
\hline 695400.74 & 3373894.43 & 2800 & 2117.42 \\
\hline 695417.69 & 3373884.87 & 3100 & 2522.08 \\
\hline 694429.25 & 3374329.77 & 700 & 1145.16 \\
\hline 694541.01 & 3374318.70 & 1250 & 1626.34 \\
\hline 694295.68 & 3374320.27 & 700 & 950.41 \\
\hline 694081.94 & 3374205.31 & 1950 & 2969.80 \\
\hline 693696.69 & 3373094.27 & 300000 & 284251.54 \\
\hline 693575.95 & 3374496.41 & 100000 & 148834.48 \\
\hline
\end{tabular}




\subsection{April 2009}

\begin{tabular}{|c|c|c|c|}
\hline Easting (m) & Northing (m) & discharge $\left(\mathrm{cm}^{3} \mathrm{sec}^{-1}\right)$ & predicted discharge $\left(\mathrm{cm}^{3} \mathrm{sec}^{-1}\right)$ \\
\hline 696577.00 & 3375064.00 & 26688 & 17558.21 \\
\hline 696515.00 & 3375060.00 & 31324 & 25683.40 \\
\hline 696526.00 & 3375081.00 & 10878 & 7960.94 \\
\hline 696378.00 & 3375075.00 & 3155 & 2196.50 \\
\hline 696374.00 & 3375047.00 & 31168 & 28808.33 \\
\hline 696312.00 & 3374949.00 & 44714 & 33750.34 \\
\hline 693684.21 & 3374490.78 & 181142 & 148233.29 \\
\hline 693857.70 & 3374480.17 & 134261 & 134367.19 \\
\hline 694237.23 & 3374550.07 & 159510 & 123111.22 \\
\hline 694371.93 & 3374575.91 & 96597 & 120688.53 \\
\hline 694445.52 & 3374574.44 & 123230 & 115351.89 \\
\hline 694706.00 & 3374606.66 & 142841 & 111689.91 \\
\hline 694808.19 & 3374666.05 & 133061 & 103032.24 \\
\hline 694815.26 & 3374674.12 & 24251 & 14558.59 \\
\hline 695449.36 & 3374792.20 & 70782 & 70543.57 \\
\hline 695317.14 & 3374776.63 & 115771 & 82714.80 \\
\hline 695400.81 & 3374783.02 & 18354 & 10476.11 \\
\hline 695613.16 & 3374808.59 & 46630 & 69195.87 \\
\hline 695756.20 & 3374863.59 & 11422 & 10024.07 \\
\hline 695787.02 & 3374851.22 & 81630 & 57339.57 \\
\hline 695914.95 & 3374827.10 & 24757 & 41590.16 \\
\hline 695922.74 & 3374822.92 & 31480 & 15071.30 \\
\hline 696011.72 & 3374871.04 & 6903 & 2472.52 \\
\hline 696019.12 & 3374873.43 & 52090 & 38588.88 \\
\hline 696127.23 & 3374876.96 & 44644 & 36223.61 \\
\hline 696267.06 & 3374905.73 & 51745 & 34747.90 \\
\hline 696335.93 & 3374970.34 & 52171 & 29296.08 \\
\hline 696577.00 & 3375064.00 & 26688 & 17558.21 \\
\hline 696515.00 & 3375060.00 & 31324 & 25683.40 \\
\hline 696526.00 & 3375081.00 & 10878 & 7960.94 \\
\hline 696378.00 & 3375075.00 & 3155 & 2196.50 \\
\hline 696374.00 & 3375047.00 & 31168 & 28808.33 \\
\hline 696346.42 & 3374960.48 & 8141 & 4287.83 \\
\hline 696916.41 & 3374703.37 & 2704 & 2773.16 \\
\hline 696913.37 & 3374697.05 & 2131 & 1230.01 \\
\hline 695406.38 & 3373894.53 & 6791 & 2117.42 \\
\hline 695284.53 & 3373820.41 & 4975 & 6209.90 \\
\hline 695268.73 & 3373828.01 & 12171 & 7245.29 \\
\hline 695207.06 & 3373539.81 & 28499 & 16435.07 \\
\hline 695163.07 & 3373472.73 & 285299 & 214562.12 \\
\hline 695825.64 & 3373844.08 & 20009 & 6515.55 \\
\hline
\end{tabular}




\begin{tabular}{cccc} 
Easting $(\mathrm{m})$ & Northing $(\mathrm{m})$ & discharge $\left(\mathrm{cm}^{3} \mathrm{sec}^{-1}\right)$ & predicted discharge $\left(\mathrm{cm}^{3} \mathrm{sec}^{-1}\right)$ \\
\hline \hline 695818.15 & 3373874.10 & 3098 & 1747.84 \\
695829.40 & 3373872.17 & 6847 & 4724.56 \\
695870.78 & 3373925.80 & 1292 & 1436.14 \\
695873.99 & 3373937.31 & 7298 & 2562.84 \\
694804.18 & 3374918.55 & 4777 & 2926.35 \\
694811.43 & 3374929.34 & 15554 & 10799.79 \\
694864.00 & 3374985.35 & 9906 & 7942.65 \\
694853.04 & 3375015.85 & 6866 & 2769.17 \\
694999.62 & 3375057.93 & 11789 & 6421.11 \\
695043.10 & 3375092.62 & 3376 & 2175.13 \\
695043.00 & 3375070.20 & 5248 & 3776.13 \\
695410.00 & 3373885.00 & 4173 & 2590.45 \\
697528.00 & 3374024.00 & 995 & 2400.35 \\
695529.00 & 3374749.00 & 685 & 490.24 \\
695437.00 & 3374602.00 & 263 & 422.30 \\
695434.00 & 3374600.00 & 10759 & 9777.40
\end{tabular}

\section{References}

S1. Abrams, D. M. et al. Growth laws for channel networks incised by groundwater flow. Nature Geoscience 28, 193-196 (2009).

S2. Hecht, F., Pironneau, O., Le Hyaric, A. \& Ohtsuka, K. Freefem++ Manual (2005).

S3. Bear, J. Hydraulics of groundwater (McGraw-Hill New York, 1979).

S4. Schorghofer, N., Jensen, B., Kudrolli, A. \& Rothman, D. H. Spontaneous channelization in permeable ground: theory, experiment, and observation. Journal of Fluid Mechanics 503, 357-374 (2004).

S5. Lobkovsky, A. E., Smith, B. E., Kudrolli, A., Mohrig, D. C. \& Rothman, D. H. Erosive dynamics of channels incised by subsurface water flow. J. Geophys. Res 112 (2007).

S6. Lamb, M. P., Dietrich, W. E., Aciego, S. M., DePaolo, D. J. \& Manga, M. Formation of Box Canyon, Idaho, by megaflood: Implications for seepage erosion on Earth and Mars. Science 320, 1067 (2008). 